\title{
Avaliação dos níveis de ansiedade dos pacientes assistidos no Serviço de Reabilitação Cardiovascular do Instituto Dante Pazzanese de Cardiologia
}

\author{
Gerson HEIDRICH*, Lourdes Helena de CAMPOS*
}

RBCCV $44205-240$

HEIDRICH, G. \& CAMPOS, L. H. - Avaliaçāo dos niveis de ansiedade dos pacientes assistidos no Serviço de Reabilitação Cardiovascular do Instituto Dante Pazzanese de Cardiologia. Rev. Bras. Cir. Cardiovasc. 9 (2): 123-128, 1994.

RESUMO: A ansiedade tem sido mencionada como um dos fatores relevantes para o desenvolvimento e manutenção das coronariopatias. Neste estudo avaliaram-se os niveis de ansiedade traço e estado de 100 pacientes assistidos pelo Serviço de Reabilitação Cardiovascular da Divisão de Diagnóstico e Terapêutica do Instituto Dante Pazzanese de Cardiologia. Submetidos à aplicaçāo do Inventário de Ansiedade TraçoEstado (IDATE) antes das atividades físicas, os pacientes que apresentaram estado de ansiedade acima da média populacional foram reavaliados após as atividades, com o objetivo de verificar se estas colaboravam para a diminuiçāo dos niveis de ansiedade estado dos mesmos. Pelos resultados obtidos, pôde-se deduzir que os pacientes se beneficiaram com este procedimento, quanto à diminuição dos niveis de ansiedade estudados. Destacaram-se, tambèm, a hostilidade e a sociabilidade caracterizando as formas de interrelações. Em nivel psicológico, os autores sugerem o uso de técnicas de relaxamento, dinâmicas de grupo e orientaçăo psicológica como recursos utilizados para mudança de comportamento e estilo de vida do indivíduo, auxiliando-o a estabelecer um melhor funcionamento de seu psiquismo, bem como o equilíbrio homeostático.

DESCRITORES: coronariopatias, análise psicológica de pacientes; coronariopatias, reabilitação.

\section{INTRODUÇÃO}

Há muitos anos as doenças das coronárias têm ocupado lugar de destaque no quadro das enfermidades. $\mathrm{O}$ aumento da incidência das coronariopatias nas sociedades modernas vêm preocupando os profissionais da saúde, bem como a população em geral. Em diversos países atribui-se a essas doenças o primeiro lugar como causa de morte.

Dos fatores já estudados sobre as possiveis causas, considerando, tanto as orgânicas e ambientais quanto as emocionais, um tem sido mencionado como relevante para o desenvolvimento e manutenção das coronariopatias: a ansiedade.

A ansiedade pode ser considerada como um estado afetivo que traz o sentimento de insegurança, em que $o$ individuo se sente ameaçado frente às situações que the escapam ao controle. Em situação de enfermidade evidenciam-se como causas - medo da morte, à invalidez crônica, incerteza sobre a doença e prognóstico, mudanças no padrão normal da vida diária, bem como a hospitalização, com restrições e limitações na dieta e nas atividades.

Trabalho realizado no Instituto Dante Pazzanese de Cardiologia. São Paulo, SP, Brasil.

Recebido para publicação em junho de 1994.

- Do Instituto Dante Pazzanese de Cardiologia e Fundação Adib Jatene.

Endereço para correspondência: Gerson Heidrich. Av. Dr. Dante Pazzanese, 500. Serviço de Reabilitaçăo. CEP: 04012-180 São Paulo, SP, Brasil. 
HEIDRICH, G. \& CAMPOS, L. H. - Avaliação dos niveis de ansiedade dos pacientes assistidos no Serviço de Reabilitação Cardiovascular do Instituto Dante Pazzanese de Cardiologia. Rev. Bras. Cir. Cardiovasc., 9 (2): 123-128, 1994.

Algumas manifestações se dão pela falta de habilidade do indivíduo de se concentrar e reter informações, inquietação e insônia, verbalização exacerbada, tremores e suor nas palmas das mãos ${ }^{21}$.

A personalidade tipo $\mathrm{A}$, descrita por FRIEDMAN \& ROSENMAN ${ }^{14}$, abordada em estudos de FREEMAN \& NIXON ${ }^{13}$ e BOOTH-KEWLEY \& FRIEDMAN ${ }^{5}$, caracteriza o coronariano como um indivíduo altamente competitivo, com necessidade constante de realizar coisas sempre no menor prazo de tempo possível. Geralmente é perfeccionista, busca êxitos profissional e social vorazmente e possui necessidade primordial de ser amado e elogiado por todos. Segundo BERSIER e BLOCH ${ }^{3}$, o mesmo apresenta dificuldade em expressar diretamente sua agressividade, exceto, às vezes, de maneira impulsiva e explosiva. Tende, também, a reprimir os próprios sentimentos, em particular a ansiedade.

Embora várias pesquisas em psicologia sejam direcionadas ao estudo de pacientes coronarianos, pouco se tem estudado sobre a dinâmica emocional dos pacientes que pertencem aos programas de reabilitação, submetidos ou não a procedimentos cirúrgicos.

Alguns estudos sobre os efeitos psicológicos dos exercícios físicos em reabilitação ${ }^{22}$ mostraram que os pacientes se beneficiaram dessas atividades: aumento do sentimento de bem-estar e superioridade; aumento de autoconfiança e motivação, porém sem diferenciaçăo no nivel de ansiedade.

Mediante observação de pacientes assistidos pelo Serviço de Reabilitação Cardiovascular do Instituto Dante Pazzanese de Cardiologia (IDPC), pôdese perceber que a ansiedade permeava as interrelações. Resolveu-se, então, avaliar quantitativa e qualitativamente os niveis de ansiedade dos pacientes assistidos pelo referido Serviço.

\section{OBJETIVO}

Avaliar quantitativa e qualitativamente os niveis de ansiedade dos pacientes assistidos pelo Serviço de Reabilitação Cardiovascular do Instituto Dante Pazzanese de Cardiologia, que mantiveram freqüência regular (acima de $75 \%$ ) nas aulas de atividades físicas, com o objetivo de verificar se estas realmente colaboram para a diminuição dos níveis de Ansiedade Estado dos mesmos.

\section{CASUISTICA E MÉTODOS}

Foram estudados 100 pacientes, sendo 88 do sexo masculino e 12 do sexo feminino, por um período de doze meses, matriculados no Serviço de Reabilitação Cardiovascular do IDPC.

Divididos em 12 grupos distintos, sendo que um deles formado por 12 pacientes do sexo feminino, verificaram-se, quantitativamente, os niveis de ansiedade dos mesmos, tendo sido submetidos à aplicação do IDATE partes I AE e II - AT, utilizandose de 15 minutos iniciais das aulas de atividades físicas, e avaliados de acordo com os seguintes níveis: a) Acima da média, b) Abaixo da média e c) Dentro da média populacional 24 .

O IDATE (Inventário de Ansiedade Traço-Estado) de SPILBERGER et alii ${ }^{24}$ é composto de duas escalas distintas de auto-relatório, elaboradas para medir quantitativamente dois conceitos de ansiedade: Estado de Ansiedade (AE) e Traço de Ansiedade (AT).

O Estado de Ansiedade tem como características a ansiedade transitória, tensão, nervosismo, preocupação, sentimentos desagradáveis de tensão e apreensão conscientes. O Traço de Ansiedade, por sua vez, apresenta tendência à ansiedade, reação a situação percebida como ameaçadora, ameaça a auto-estima e situações que envolvem relações inter-pessoais.

Posteriormente à análise quantitativa resultante da primeira avaliação, os pacientes que apresentaram Estado de Ansiedade acima da média populacional foram submetidos à reaplicação do IDATE parte I - AE, após atividades físicas, para verificar se haveria ou não variação do nivel de ansiedade dos mesmos.

Verificou-se, também, qualitativamente, através de observação, a dinâmica de grupo dos pacientes assistidos pelo referido Serviço de Reabilitação.

\section{RESULTADOS}

Dos 100 pacientes estudados, 88 do sexo masculino e 12 do sexo feminino, chegou-se ao seguinte quadro após avaliação do IDATE parte I AE e parte II - AT (Tabela 1).

Dados quantitativos, constantes da Tabela 1, mostraram que em $76 \%$ dos pacientes assistidos pelo Serviço de Reabilitação Cardiovascular do IDPC os níveis de Estado de Ansiedade estavam dentro da média populacional. Dos pacientes avaliados, $20 \%$ apresentaram $\mathrm{AE}$ acima da média e $4 \% \mathrm{AE}$ abaixo da média. A análise do Traço de Ansiedade dos pacientes assistidos pelo referido Serviço indicou que $53 \%$ estavam dentro da média, $46 \%$ acima da média e $1 \%$ abaixo da média populacional. 0 grupo formado por pacientes do sexo feminino não 
HEIDRICH, G. \& CAMPOS, L. H. - Avaliação dos niveis de ansiedade dos pacientes assistidos no Serviço de Reabilitação Cardiovascular do Instituto Dante Pazzanese de Cardiologia. Rev. Bras. Cir. Cardiovasc., 9 (2): 123-128, 1994.

TABELA 1

\begin{tabular}{lcccrrr}
\hline IDATE & \multicolumn{2}{c}{ MASCULINO $\%$} & \multicolumn{2}{c}{ FEMININO \% } & \multicolumn{2}{c}{ TOTAL\% } \\
MÉdIA POP. & AE & AT & AE & AT & AE & AT \\
\hline Acima & 22,7 & 46,6 & 0 & 41,7 & 20,0 & 46,0 \\
Abaixo & 2,3 & 0 & 16,7 & 8,3 & 4,0 & 1,0 \\
Média & 75,0 & 53,4 & 83,3 & 50,0 & 76,0 & 53,0 \\
\hline
\end{tabular}

AE: Estado de Ansiedade

AT: Traço de Ansiedade

apresentou Estado de Ansiedade acima da média populacional.

De 20 pacientes que apresentaram Estado de Ansiedade acima da média populacional foi possível reavaliar 10 , sendo os mesmos submetidos à reaplicação do IDATE parte I - AE, após atividades físicas. Dos pacientes não reavaliados, 6 foram excluidos deste estudo por não participarem regularmente das atividades propostas pelo Serviço de Reabilitação, 3 por desistência do referido Serviço e 1 por óbito.

Dados quantitativos, constantes da Tabela 2, mostraram que, após reavaliação do Estado de Ansiedade, 5 pacientes apresentaram $A E$ dentro da média e 5 acima da média populacional.

Destacaram-se, qualitativamente, a hostilidade e a sociabilidade caracterizando as formas de interrelações dos pacientes estudados.

\section{COMENTÁRIOS}

Pelos resultados do presente trabalho, comparando-se os níveis de ansiedade Traço (46\%) e Estado $(20 \%)$ acima da média populacional, pôdese deduzir que os pacientes assistidos pelo Serviço de Reabilitação Cardiovascular do IDPC têm-se beneficiado com as aulas de atividades físicas realizadas, quanto à diminuição dos níveis de ansiedade estudados.

A reavaliação dos pacientes que apresentaram Estado de Ansiedade acima da média reforça a

TABELA 2

\begin{tabular}{lc}
\hline IDATE & PACIENTES \\
MÉDIA POP. & REAVAL. - AE \\
\hline Acima & 05 \\
Abaixo & 0 \\
Média & 05 \\
\hline Total & 10 \\
\hline
\end{tabular}

AE: Estado de Ansiedade dedução mencionada, pois a diminuição do Estado de Ansiedade foi verificada em $50 \%$ dos pacientes deste grupo, após as atividades físicas, atingindo, assim, um nivel de ansiedade dentro da média populacional.

Um fator observado que se evidenciou e caracterizou os grupos foi a hostilidade. Pressupõe-se, neste contexto, a reação agressiva de determinados individuos mediante provocação, ou em estado de competição ${ }^{26}$. Utilizam-se, por vezes, do humor como forma branda de agressão, ou, mesmo, como possivel negação às situações de conflitos, encobrindo, assim, a ansiedade que marcou as interrelaçōes nos grupos.

A negação, talvez o mais importante mecanismo de defesa, tem sido apontada por alguns autores como fundamental no processo de adaptação a situaçōes vividas, pois diminui a ansiedade durante a fase inicial da doença ${ }^{11}$. Sendo uma característica da hostilidade, o que pôde ser percebido é que este mecanismo de defesa tem como uma das funções conservar a imagem que o indivíduo faz de si mesmo, mantendo o domínio sobre suas ações e expectativas de vida. É um controle psicológico que o individuo utiliza para ordenar sua própria vida.

No entanto, o uso do mecanismo de negação pode acarretar uma ansiedade ainda maior que a esperada, pois o individuo ao negar seus próprios temores e sua situaçāo de desconforto, dificulta a sua própria elaboração psiquica quanto aos conflitos. A elaboração psíquica das situaçōes de conflitos por parte do indivíduo é de fundamental importância para que o mesmo, mediante a conscientização de sua real situação, possa vivenciá-la e, assim, buscar a diminuição dos niveis de ansiedade, bem como as possiveis resoluçōes.

Durante o periodo de observação dos grupos houve o óbito de um dos pacientes. A reação de grande parte de seus colegas reforçou as citações sobre a hostilidade, pois, embora tenha havido uma manifestação de pesar, algumas brincadeiras e gozações se fizeram presentes.

Em seu aspecto psicológico, o indivíduo pode negar a realidade de sua morte por algum tempo. 
HEIDRICH, G. \& CAMPOS, L. H. - Avaliação dos niveis de ansiedade dos pacientes assistidos no Serviço de Reabilitaçāo Cardiovascular do Instituto Dante Pazzanese de Cardiologia. Rev. Bras. Cir. Cardiovasc., 9 (2): 123-128, 1994.

Inconscientemente, não pode conceber a sua própria morte, o que o leva a reforçar sua crença na imortalidade. $O$ indivíduo tem a necessidade de se defender contra o medo da morte e contra a incapacidade de prevê-la. Tenta, assim, de várias formas, precaver-se dessa realidade ${ }^{18}$. A morte é, e talvez sempre será, um fator de angústia para o ser humano. Ela é um fato e, portanto, uma desencadeadora de ansiedade quando da iminência de sua possibilidade.

Segundo a literatura, a cultura ocidental nega a morte. Tem o pólo vida como bom e o pólo morte como ruim, desconsiderando que a vida é permeada pela dialética dessas polaridades. É justamente isso que nos ajuda a compreender porque tantas pessoas, tantos profissionais de ajuda - principalmente os da área da saúde - não lidam muito bem com a morte. Nos hospitais, a morte é tida como uma inimiga a ser vencida e não como o fim natural da vida ${ }^{25}$.

Um outro fator que se evidenciou e caracterizou os grupos foi a sociabilidade. Uma sociabilidade que favoreceu o bem-estar dos pacientes, propiciando integração satisfatória e o bom andamento das aulas de atividades físicas. Pouco se falou sobre doenças ou conflitos específicos. Os assuntos discutidos nos grupos praticamente estavam associados às questões sociais cotidianas, tais como: política, economia e esportes.

A prática do volleyball, que se dava uma vez por semana, nos quinze minutos finais das aulas, foi a mais apreciada pelos pacientes. Esta atividade era solicitada com freqüência pelos mesmos, e o que chamou a atenção foi a não competitividade aparente, contrapondo-se, assim, uma das principais características do padrão de comportamento tipo A do coronariano, que é o de um indivíduo altamente competitivo ${ }^{5}$. As disputas eram dotadas de caráter amistoso, embora com o cuidado de não se cometer erros. Não se contavam pontos e, portanto, nāo havia vencedores, o que pareceu favorecer a descontração dos pacientes. Porém, devese considerar a hipótese de que esta prática era solicitada com veemência, justamente pelo caráter de competitividade de uma atividade desportiva.

Pode-se dizer, portanto, que os fatores psicossociais observados são importantes no tratamento e prevenção de doenças coronarianas. O meio social competitivo, apressado e de baixa coesão grupal são agentes desencadeadores de ansiedade. Por sua vez, a ansiedade favorece condutas inadequadas, tais como: sedentarismo, tabagismo e dietas polissaturadas. Esses comportamentos, já apontados em diversos estudos, sāo, assim, fatores responsáveis pelo desenvolvimento das coronariopatias ${ }^{9}$.
Os suportes sociais que, segundo $\operatorname{COBB}^{9}$, são todas as estruturas grupais que propiciam ao indivíduo sentir-se amado e valorizado, cuidado e protegido e membro de uma rede de interaçōes e comunicaçōes que funciona franca, clara e solidária, são fundamentais na prevenção e recuperação da dinâmica emocional e estrutural do mesmo. O modo pelo qual o paciente vive sua doença também e. no contexto estudado, primordial para o seu bom funcionamento psiquico. Em entrevistas, percebeuse que grande parte dos mesmos conhecia muito pouco de sua doença. Questionados, justificaramse pela falta de comunicação na relação médicopaciente e pelo próprio estado de ansiedade frente à situação vivenciada.

Ao se falar na relação médico-paciente, considerando-se desde o primeiro contato e principalmente em situação de hospitalização, torna-se inevitável a explanaçāo de dois fenômenos: transferência e contratransferência. A transferência, especialmente em estado regressivo do paciente, faz com que o mesmo atue com o seu médico do mesmo jeito que atuava com importantes figuras do seu passado. Dessa forma, o médico pode ser visto como uma figura positiva e acolhedora ou negativa e desinteressada. É justamente este fenômeno que explica o fato de o médico se defrontar com pacientes que desenvolvem dependência, e aqueles que apresentam hostilidade com negativismo em colaborar, ou, mesmo, reconhecer melhoras em seu quadro.

Por outro lado, segundo ZIMERMAN \& MELLO FILHO ${ }^{27}$, o médico também tem sentimentos em relação a seus pacientes, fenômeno este denominado contratransferência. Pode ser decorrente de uma resposta do médico às angústias nele depositadas pelo paciente, ou uma transferência do mesmo em relação a seu paciente, pelo fato de este estar lhe representando uma figura importante.

Estes dois fenômenos são simultâneos e interativos, e portanto, imprescindíveis na relação médico-paciente. O profissional conhecedor da dinâmica destes fenômenos pode fazer uso adequado dos mesmos, aprimorando sua comunicação com o paciente ao saber ver e escutar; ao evitar o que BALINT ${ }^{1}$ chama "função apostólica", que é a tendência de catequizar os pacientes com julgamentos morais e padrōes de comportamento, bem como a necessidade de mostrar que é um profissional capaz de ajudar; e ao fazer uso de uma linguagem acessivel ao paciente, para que o mesmo saiba o que está the acontecendo e ambos possam lidar, conhecidos o diagnóstico e prognóstico, com a verdade.

É comum ouvir dos profissionais de saúde que o paciente deve ser assistido em seu todo, pois o mesmo tem dois lados: o físico e o emocional. É 
HEIDRICH, G. \& CAMPOS, L. H. - Avaliação dos niveis de ansiedade dos pacientes assistidos no Serviço de Reabilitação Cardiovascular do Instituto Dante Pazzanese de Cardiologia. Rev. Bras. Cir. Cardiovasc., 9 (2): 123-128, 1994.

comum também, ouvir que há a necessidade de um trabalho multiprofissional, cujo objeto maior é a recuperação e o bem-estar do paciente. No entanto, cabe chamar a atenção ao aspecto do bom funcionamento da equipe multiprofissional. Por vezes, devido a falta de reuniões periódicas e discussão de casos, alicerçadas a uma comunicação inadequada, vê-se prejudicada quanto à congruência. Esta. por sua vez, é essencial para a interação e manutenção da equipe, que deve ter como objetivo assistir o paciente em seu aspecto global, isto é, biopsicossocial.

\section{CONCLUSÃO}

Pode-se concluir, neste trabalho, que as aulas de atividades físicas colaboraram para a diminuição dos níveis de ansiedade estudados, bem como propiciaram interação satisfatória e boa sociabilidade, o que é muito importante para prevençăo e recuperação da dinâmica emocional dos pacientes.

Todavia, para melhorar os niveis de ansiedade e, conseqüentemente, a qualidade de vida dos pacientes do Programa de Reabilitação do IDPC, há a necessidade de se desenvolver um trabalho em equipe multiprofissional, o que é uma proposta do Serviço de Reabilitação Cardiovascular do Instituto, sem, no entanto, desconsiderar os aspectos da congruência.

Em nivel psicológico, os autores sugerem o uso de técnicas de relaxamento, dinâmicas de grupo e orientação psicológica como recursos utilizados para mudança de comportamento e estilo de vida do individuo, auxiliando-o a estabelecer um melhor funcionamento de seu psiquismo, bem como o equilíbrio homeostático.

RBCCV 44205-240

HEIDRICH, G. \& CAMPOS, L. H. - Evaluation of the levels of anxiety on patients assisted by the Service of Cardiovascular Rehabilitation from the Diagnosis and Therapeutic Division of Instituto Dante Pazzanese de Cardiologia. Rev. Bras. Cir. Cardiovasc., 9 (2): 123-128, 1994.

ABSTRACT: Anxiety has been mentioned as one of the relevant agents for the development and maintenance of coronary artery disease. In this study were evaluated the levels of State - Trait Anxiety of 100 patients assisted by the Service of Cardiovascular Rehabilitation of Instituto Dante Pazzanese de Cardiologia, São Paulo, Brasil. Patients were submited to the State-Trait Anxiety Inventory-STAl before physical activities, those who showed State Anxiety above the average were revaluated after the activities, to verify if the activities helped to diminish the levels of State Anxiety. The conclusion given from the results was that the patients benefited from this procedure, considering the decrease of anxiety levels studied. Hostility and sociability also stood out as ways of interrelations. Psychologicaly speaking, the authors suggest that the use of relaxing technics, group dynamics and psychological guidance as means to change the behavior and the life style of an individual, help to establish a better psychism functioning as well as a homeostasis balance.

DESCRIPTORS: coronary diseases, psicological profile of patients; coronary diseases, rehabilitation.

\section{REFERÊNCIAS BIBLIOGRÁFICAS}

1 BALINT, M. - O Médico, seu paciente e a doença. Rio de Janeiro, Atheneu, 1984.

2 BENJAMIN, A. - A Entrevista de Ajuda. 4. ed. São Paulo, Martins Fontes, 1986.

3 BERSIER, A. L. \& BLOCH, A. - Le rôle du psychiatre dans une Unité de Soins Intensifs. Méd. Hyg. (Genebra), 34: 1609-1610, 1976.

4 BLEGER, J. - Psico-higiene e psicologia institucional. Porto Alegre, Artes Médicas, 1984.
5 BOOTH-KEWLEY, S. \& FRIEDMAN, H. S. - Personality, type A behavior and coronary heart disease: the role of emotional expression. J. Person. Soc. Psychol., 53: 783-792, 1979

6 BRAIER, E. A. - Psicoterapia: breve de orientaçāo psicanalítica. Sāo Paulo, Martins Fontes, 1986.

7 CAMPOS, L. H. \& NASCIMENTO, G. F. - Traços psicológicos dos pacientes submetidos a angioplastia transluminal coronária. Rev. Bras. Cir. Cardiovasc., 5: 195-199, 1990.

8 CASSORLA, R. M. S. (coord.) - Da Morte: estudos brasileiros. São Paulo, Papirus, 1991. 
HEIDRICH, G. \& CAMPOS, L. H. - Avaliação dos niveis de ansiedade dos pacientes assistidos no Serviço de Reabilitação Cardiovascular do Instituto Dante Pazzanese de Cardiologia. Rev. Bras. Cir. Cardiovasc., 9 (2): 123-128, 1994.

9 СОBB, S. - Social support a moderator of life stress. Psychother. Med., 38: 300-314, 1976.

10 FARDY, P. S.: YANOWITZ, F. G.; WILSON, P. - Cardiac rehabilitation adult fitness, and exercise testing. 2. ed. Philadelphia, Lea \& Febiger, 1988. p. 372-378.

11 FINE, R. - Troubled men: the psychology emotional conflicts, and therapy of men. San Francisco, Jossey Bass, 1988. p. 34.

12 FOUCAULT, M. - Microfisica do Poder. 10. ed. Rio de Janeiro, Graal, 1992.

13 FREEMAN, I. J. \& NIXON, P. G. F. - The effect of the type $A$ behaviour pattern on myocardial ischemia during daily life. Int. J. Cardiol., 17: 145-154, 1987.

14 FRIEDMAN, M. \& ROSENMAN, R. H. - Associaton of specific overt behavior pattern with blood and cardiovascular findings. JAMA, 169: 1286-1296, 1959.

15 GUIRADO, M. - Psicologia institucional. São Paulo, EPU, 1987.

16 HAYNAL, A. \& PASINI, W. - Manual de medicina psicossomática. São Paulo, Masson, 1983.

17 JAFFĖ. A.; FREY-ROHN, L.; FRANZ, M. L. V. - A Morte à luz da psicologia. São Paulo, Cultrix, 1989.

18 KÜBLER-ROSS, E, - Sobre a morte e o morrer. 3, ed. São Paulo, Martins Fontes, 1987.
LAMOSA, B. W. R. - Psicologia aplicada à cardiologia. São Paulo, Fundo Editorial Byk, 1990.

LAMOSA, B. W. R.; QUADROS, C. S. O.; ZAMBON, E. - Ansiedade em cardiopatas cirúrgicos: um estudo da variaçāo do pré e pós-operatório. Rev. Bras. Med. (Cardiologia), 4 (2): abril, 1985

MELLO FILHO, J. (ed.) - Psicossomática hoje. Porto Alegre, Artes Médicas, 1992.

ONGARO, S. - O Paciente coronariano em reabilitação: uma abordagem psicossomática. Rev. SOCESP, 1 : $9-16,1991$.

23 RAMOS, D. G. - A Psique do coração: uma leitura analítica do seu simbolismo. São Paulo, Cultrix, 1990.

SPIELBERGER, C. D.: GORSUCH, R. L.; IUSHENE, R. E. - Inventário de Ansiedade Traço-Estado - IDATE: manual de psicologia aplicada. (Tradução de Angela Biaggio e Luis Natalicio), CEPA, Rio de Janeiro, 1979.

VARGAS, N. S. - A Morte como simbolo de transformação. Anais do $1^{\circ}$ Congresso Brasileiro de Psiquiatria e Medicina Interna. Sāo Paulo, 1987. p. 131-134.

26 WRIGHT. L. - The Type A behaviour patterns and coronary artery disease. Am. Psych., 43: 2-4, 1988.

27 ZIMERMAN, D. E. \& MELLO FILHO, J. - Psicossomática hoje: a formação psicológica do médico. Porto Alegre, Artes Medicas, 1992. p. 64-69. 